\title{
Unidade amostral para pulgão em algodoeiro e relação com o número de plantas de atacadas
}

\author{
Cotton aphid sample unit and its relationship with the number \\ of attacked plants
}

'Universidade da Integração Internacional da Lusofonia AfroBrasileira, Redenção, CE, Brasil 2Universidade Federal do Ceară, Fortaleza, CE, Brasil

${ }^{3}$ Instituto Federal do Ceară, Crateús, CE, Brasil

Universidade Federal do Sul da Bahia

- UFSB, Porto Seguro, BA, Brasil

* autor correspondente

$\boldsymbol{\nabla}$ gutemberg.moraes@unilab.edu.br

João Gutemberg Leite Moraes ${ }^{1 *}$, Ervino Bleicher ${ }^{2}$, Jefté Ferreira da Silva ${ }^{3}$,

Gleidson Vieira Marques ${ }^{4}$

RESUMO: O pulgão do algodoeiro, Aphis gossypii Glover (Hemiptera: Aphididae), é uma das mais sérias pragas sugadoras do algodoeiro. Conduziu-se a presente pesquisa com o objetivo de determinar um local de amostragem para o pulgão, considerando o perfil vertical da planta do algodoeiro, bem como estudar a relação entre o número de colônias e o percentual de plantas atacadas. Realizaram-se experimentos em condições de campo, no Campus do Pici, da Universidade Federal do Ceará, em Fortaleza, e na Fazenda Experimental Lavoura Seca, em Quixadá, CE. Para o estudo da distribuição vertical do pulgão, adotou-se uma metodologia de escala de notas, que variava de 0 a 3 , para as três folhas totalmente expandidas do ápice em cada planta. Neste estudo avaliavam-se 10 plantas por parcela, em um total de 21 parcelas. Para o estudo da relação entre o número de colônias e a percentagem de plantas atacadas, procedeu-se à contagem do número de colônias por planta, em até 16 plantas por parcela, em 25 parcelas. Observou-se que a primeira folha completamente expandida, partindo-se do ápice para a base, pode ser adotada como unidade amostral para o pulgão em algodoeiro. A relação entre o número de colônias de pulgão e a percentagem de plantas atacadas demonstrou que há um ajuste ao modelo de regressão linear.

PALAVRAS-CHAVE: Aphis gossypii, insecta, MIP, unidade amostral.
ABSTRACT: The cotton aphid Aphis gossypii Glover (Hemiptera: Aphididae) is one of the most dangerous sucking pests of cotton. The present study aimed to determine sample unit, based on within-plant distribution, as well as the relationship between number of colonies on the plant and the actual percentage of infested plants. Field experiments were performed under natural infestation, at Fazenda Lavoura Seca, in Quixadá, State of Ceará (Brazil), and at the Agronomy Department of de Universidade Federal do Ceará, in Fortaleza, State of Ceará (Brazil), during the years of 2009 and 2011. The vertical distribution study used a score scale from 0 to 3, sampling the completely expanded upper tree leaves. We evaluated 10 plants per plot, of a total of 21. To study the relationship between number of insect colonies per plant and percentage of infested plants, all colonies of the plant were counted on up to 16 plants per plot, totalizing 25 plots. We observed that the first upper leave could be used as sampling unit for cotton aphid. Linear regression model represents the relationship between numbers of colonies per plant and actual percentage of cotton plant infested by aphid.

KEYWORDS: Aphis gossypii, insect, IPM, sampling unit.

\section{Introdução}

Dentre os fitófagos-pragas do algodoeiro herbáceo (Gossypium hirsutum L. raça latifolium Hutch.), o pulgão Aphis gossypii Glover (Hemiptera: Aphididae) destaca-se entre as pragas sugadoras (BARROS et al., 2007), pois, devido ao seu hábito alimentar, o inseto provoca danos diretos (sucção da seiva e injeção de toxinas) e indiretos (mela, fumagina 
e viroses) (ALCÂNTARA et al., 2010; FURTADO et al., 2009; LI et al., 2013; MICHELOTTO; BUSOLI, 2007; TAKIMOTO et al., 2009).

O pulgão é tido como praga inicial em algodoeiro (COSTA et al., 2010), mas o seu período crítico pode se estender para além dos 60 dias da emergência. Caso o manejo da praga e os aspectos nutricionais da cultura não ocorram de forma adequada, o período crítico se estenderá à fase de aparecimento dos capulhos (BARROS et al., 2007; BORTOLI et al., 2008).

Embora existam pesquisas visando ao controle alternativo do afídeo (ANDRADE et al., 2012; ANDRADE et al., 2013; BORTOLI et al., 2015; SUJII et al., 2008) em algodoeiro, tem-se observado, frequentemente, que o uso dos agroquímicos como única ferramenta de controle está dificultando o manejo da praga. Isto por selecionar indivíduos com resistência a esses produtos, provocar a sua ressurgência e favorecer o escape dos afídeos de seus agentes de controle natural, pela morte destes em decorrência da utilização massiva de inseticidas sintéticos, em sua maioria, não seletivos (PESSOA et al., 2004).

Conforme Silva et al. (2005), as informações como o conhecimento do artrópode-praga, o nível de controle e uma correta amostragem são aspectos básicos para o Manejo Integrado de Pragas (MIP) desta malvácea. Para uma correta amostragem, é importante definir a unidade amostral, obtida a partir do conhecimento da distribuição vertical da praga na planta.

As informações encontradas na literatura científica são contraditórias, pois evidenciam-se diferentes locais onde a praga coloniza (SILVA et al., 2005). Tal fato poderá levar a erros na definição da unidade amostral, já que esta deve ser sempre associada à fenologia da cultura. Além disso, a maioria dos trabalhos citados condiciona a avaliação da intensidade de ataque à contagem dos indivíduos. Este fato torna o processo lento, por se tratar de indivíduos pequenos e numerosos, o que pode comprometer a rapidez na decisão de efetuar ou não o controle da praga.

Para diversos fitófagos, sobretudo para aqueles de tamanho diminuto, pesquisas têm apontado que o método da análise binomial, ou método presença-ausência, torna o processo mais rápido, já que leva em conta se o indivíduo está presente ou não, independentemente do seu número (GOUVEA et al., 2007). Uma opção viável seria verificar se há relação entre o número de indivíduos por planta/estrutura e o percentual de plantas/estruturas infestadas. Esta relação já foi estudada para algumas pragas e culturas, como mosca-branca, ácaros e predadores em algodão (WILSON et al., 1983), mas em relação ao pulgão em algodoeiro não foram encontradas informações.

Diante do exposto, tiveram-se com o trabalho os seguintes objetivos: 1) Estabelecer uma unidade amostral baseada na distribuição vertical do pulgão $A$. gossypii, no intuito de fornecer subsídios para a elaboração de um plano de amostragem para o manejo do afídeo; 2) Determinar a relação entre o número de colônias do pulgão do algodoeiro e a percentagem de plantas infestadas pela praga.

\section{Material e Métodos}

\section{Estudo da unidade amostral para A. gossypii em algodoeiro}

Conduziu-se o estudo em área experimental do Departamento de Fitotecnia, Campus do Pici, da Universidade Federal do Ceará (UFC), em Fortaleza, Ceará, localizada a $3^{\circ} 44^{\prime}$ de latitude sul e $38^{\circ} 34^{\prime}$ de longitude oeste. A semeadura ocorreu no dia 30 de março de 2010, utilizando a cultivar BRS Aroeira. A referida cultivar possui resistência a viroses (TORRES et al., 2007). A área destinada às avaliações correspondeu a quinze linhas de trinta e quatro metros de comprimento, com um metro entre linhas e vinte centímetros entre plantas, sendo as linhas divididas em seis segmentos de cinco metros lineares e apenas um com quatro metros lineares, correspondendo a uma área total de $476 \mathrm{~m}^{2}$. As parcelas eram delimitadas por fitas náilon e piquetes.

Para esse experimento, as avaliações ocorreram semanalmente durante o período de 08/05/2010 a 31/05/2010, totalizando quatro avaliações, dos 39 aos 60 dias após o plantio (DAP). O delineamento experimental foi o inteiramente casualizado, com três tratamentos, representados pelas folhas e 21 repetições. Eram avaliadas as três primeiras folhas totalmente expandidas (ápice para a base), do ramo principal, em dez plantas, ao acaso, nas três fileiras centrais de cada parcela (área útil), o que correspondia a 630 observações. Para a avaliação, elaborou-se a seguinte escala de notas, que variava de 0 a 3 , onde os valores da escala obedeceram a critérios de incremento da densidade populacional, discriminadas a seguir: Nota " 0 " - ausência de pulgão; Nota "1" - a partir de um pulgão áptero ou alado; Nota "2" - até duas colônias formadas; Nota "3" - mais de duas colônias formadas. As notas obtidas pela escala foram transformadas para $\sqrt{x+0,5}$ e, posteriormente, foram submetidas à análise de variância. Aplicou-se o teste de Tukey para a comparação das médias. O manejo da área restringiu-se às capinas normais e à adubação, conforme a recomendação para a cultura, sem aplicação de inseticidas.

\section{Estudo da relação entre o número de colônias de pulgão por planta e a percentagem de plantas infestadas}

O experimento foi realizado na Fazenda Experimental Lavoura Seca da UFC, em Quixadá-CE, localizada a $4^{\circ} 59^{\prime}$ de latitude sul e $39^{\circ} 01^{\prime}$ de longitude oeste. Procedeu-se à semeadura da cultivar BRS Aroeira no dia 5 de março de 2009, no espaçamento de $0,5 \mathrm{~m} \times 0,5 \mathrm{~m}$. A área foi composta por vinte e cinco parcelas ilhadas ou quadrantes de quatro metros quadrados, compreendendo uma área total de $484 \mathrm{~m}^{2}$. O experimento foi conduzido em condições de sequeiro, sob infestação natural.

As avaliações ocorreram no período de 25/03/2009 a 29/04/2009 (dos 20 aos 55 DAP), com uma frequência semanal. Procederam-se às inspeções em até 16 plantas de algodoeiro por parcela, onde eram contadas as colônias do A. gossypii por planta. Pela literatura, uma colônia de pulgões corresponde a um conjunto que varia de quinze a vinte indivíduos (SILVA et al., 2007; SPRENKEL, 2008). Entretanto, para efeito de contagens, considerou-se a presença de uma fêmea adulta áptera, rodeada 
de suas ninfas, como representativa de cada colônia. Embora existam vários modelos matemáticos para relacionar a proporção de plantas/regiões atacadas e o número de indivíduos nesses hospedeiros, optou-se pela análise de regressão, a exemplo de Smith e Shepard (2004), a fim de verificar uma possível relação entre o número de colônias do afídeo por planta e o percentual de plantas infestadas. Neste estudo utilizou-se, para a análise de regressão, o programa eletrônico Microsoft Office Excel 2010®.

\section{Resultados e Discussão}

Em virtude da baixa incidência da praga, observada aos 60 DAP, devido à ação de predadores, optou-se por considerar apenas as análises aos 39, 46 e 53 DAP. Os resultados da análise de variância referente às notas atribuídas ao ataque do A. gossypii, nas três primeiras folhas da planta e em três datas de avaliação, estão contidos Tabela 1 . Conforme pode ser constatado, o teste $\mathrm{F}$ foi significativo $(\mathrm{p}<0,05)$ para a variável analisada, demonstrando que houve diferença em todos os períodos estudados. Isto indica que houve distribuição diferencial da praga nas folhas avaliadas.

Diante da confirmação da existência de diferença entre folhas para a densidade de pulgões, procedeu-se ao teste de médias para discriminá-las (Tabela 2).

Levando-se em conta as três datas de avaliação, a primeira folha totalmente expandida do ápice da planta sempre apresentou a maior densidade de insetos, às vezes, acompanhada das outras.

Nesta pesquisa, os resultados oriundos das avaliações, do número de colônias de pulgões em relação ao percentual de plantas infestadas, estão expressos na Figura 1.

Observa-se que o ajuste ao modelo linear, avaliado pela análise da regressão entre a proporção de plantas infestadas estimadas e observadas foi significativo $(\mathrm{p}<0,01)$, explica em quase $97,46 \%$ a variação do modelo, indicando que a proporção de plantas infestadas estimadas pode ser usada como indicador do número de colônias de pulgão por planta.

A contagem direta de $A$. gossypii foi utilizada por Calcagnolo e Sauer (1954), que realizaram avaliações em algodoeiro com auxílio de lupa, numa área de $5 \mathrm{~cm}^{2}$ na quarta folha da planta, método este certamente bastante demorado. Entretanto, Araujo (2009) considera a contagem como método viável para compor um plano de amostragem dessa praga em algodoeiro. Cabe ressaltar que a amostragem para fins de manejo necessita de rapidez, dentro de limites de precisão aceitáveis, podendo o processo implicar diretamente no aumento de custos para o produtor (PEDIGO; RICE, 2009).

A preferência de localização do $A$. gossypii nas regiões apicais do algodoeiro pode ser explicada pela distribuição diferencial de fotoassimilados na planta, onde a cada estádio fenológico ocorrem alocações direcionadas às regiões de maior demanda (FURTADO et al., 2007), associado, possivelmente, à facilidade encontrada pelos afídeos em inserir seus estiletes para reconhecer e iniciar o processo de alimentação em seu hospedeiro.

A escolha do hospedeiro (POWELL et al., 2006) e a sua aceitação (MULLINS; BURMESTER, 2010) podem ser uma
Tabela 1. Análise de variância referente às notas atribuídas ao pulgão Aphis gossypii, em três folhas de algodoeiro e em três avaliações aos 39,46 e 53 DAP.

\begin{tabular}{ccccc}
\hline \multirow{2}{*}{$\begin{array}{c}\text { Causas da } \\
\text { variação }\end{array}$} & GL & \multicolumn{3}{c}{ Q.M } \\
\cline { 3 - 5 } & & 39 & 46 & 53 \\
\hline Folha & 2 & $6,1590 * *$ & $3,2694^{*}$ & $2,8249 * *$ \\
Resíduo & 60 & 0,5369 & 0,9863 & 0,3861 \\
Total & 62 & - & - & - \\
CV $(\%)$ & & 30,96 & 40,56 & 28,64 \\
\hline
\end{tabular}

*Significativa a $5 \%$ de probabilidade $(\mathrm{p}<0,05)$. ${ }^{*}$ Significativa a $1 \%$ de probabilidade $(\mathrm{p}<0,01)$, no teste F. Q.M. = Quadrado Médio.

Tabela 2. Valores médios ${ }^{1}$ da distribuição vertical do pulgão Aphis gossypii em folhas de algodoeiro herbáceo, segundo escala de notas, em três avaliações.

\begin{tabular}{cccc}
\hline $\begin{array}{c}\text { Ordem das folhas no } \\
\text { eixo principal }\end{array}$ & \multicolumn{3}{c}{ Dia após o plantio } \\
\cline { 2 - 4 } & 39 & 46 & 53 \\
\hline Primeira & $2,94 \mathrm{a}^{2}$ & $2,82 \mathrm{a}$ & $2,53 \mathrm{a}$ \\
Segunda & $2,29 \mathrm{~b}$ & $2,49 \mathrm{ab}$ & $2,18 \mathrm{ab}$ \\
Terceira & $1,87 \mathrm{~b}$ & $2,04 \mathrm{~b}$ & $1,80 \mathrm{~b}$ \\
Média Geral & 2,37 & 2,45 & 2,17 \\
\hline
\end{tabular}

${ }^{1}$ Para fins de análise, os dados foram transformados em $\sqrt{x+0,5} \cdot{ }^{2}$ As médias na coluna não seguidas da mesma letra diferem estatisticamente entre si pelo teste de Tukey a $5 \%$ de probabilidade $(\mathrm{p}<0,05)$.

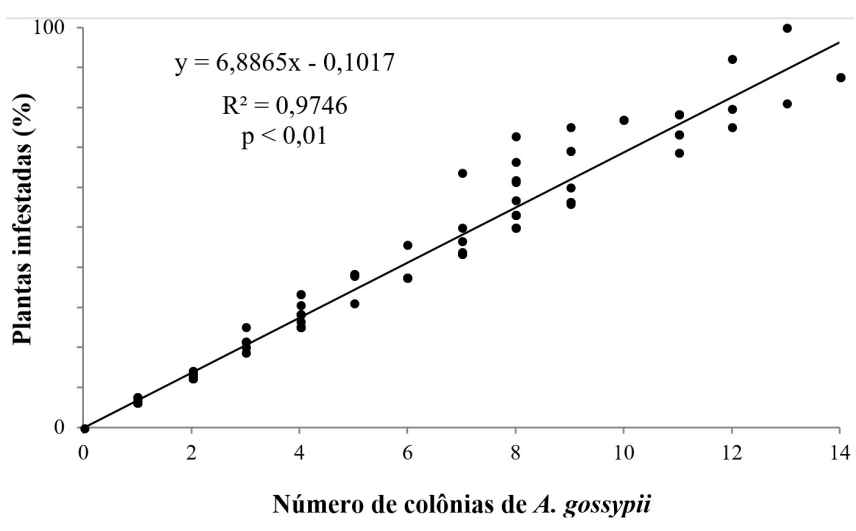

Figura 1. Relação entre o número de colônias de Aphis gossypii (eixo X) e a percentagem de plantas de algodão atacadas (eixo Y).

explicação plausível para que, nesta pesquisa, a primeira folha tenha apresentado maior densidade de insetos.

Por outro lado, considerar a divisão do algodoeiro em estratos (e.g., superior, mediano e inferior) para fins de manejo, conforme visto em algumas pesquisas (FERNANDES et al., 2011; FERNANDES et al., 2012; SILVA et al., 2005), pode causar confusão entre avaliadores e nem sempre ser praticável para todos os estádios de desenvolvimento da cultura. Dessa forma, a proposta de se tomar a primeira folha completamente expandida, como unidade amostral, possui uma maior viabilidade de utilização, já que é de fácil visualização e acesso às plantas em campo.

Em estudos observando infestações do ácaro Oligonychus yortesi, em erva-mate, foram requeridas vinte amostras quando a 
densidade média da praga atingiu 25 indivíduos por folha, considerando um nível de $10 \%$ de precisão (GOUVEA et al., 2007). Os autores avaliaram essa informação como de grande potencial para aplicação no manejo do acarino.

No manejo de $A$. gossypii, para cultivares resistentes a viroses, são considerados níveis de controle $30 \%$ a $70 \%$ de plantas infestadas (SANTOS, 2007), o que corresponderia a 4,3 e 10,2 colônias de $A$. gossypii, por planta. Por outro lado, foi verificado por Calcagnolo e Sauer (1954) que uma densidade média de 10,72 afídeos $/ \mathrm{cm}^{2}$ de folha, ou um total de 75,07 afídeos $/ \mathrm{cm}^{2}$, durante 49 dias, reduziram a produção em $44,1 \%$. Da mesma forma, estes autores verificaram que, em média, 4,4 pulgões $/ \mathrm{cm}^{2}$, ou o acumulado de 30,81 afídeos $/ \mathrm{cm}^{2}$, no mesmo período, causaram um decréscimo de $16,5 \%$ na produção.

O modelo obtido pelo presente estudo pode direcionar a tomada de decisão para efeitos de adoção de medidas de controle do pulgão do algodoeiro, inclusive podendo ser associado ao processo de amostragem sequencial da praga nesta cultura.

Considerando-se os resultados aqui relatados, pode-se orientar melhor os avaliadores, definindo-se a primeira folha totalmente expandida do ápice como unidade amostral. O tempo gasto na amostragem pode ser diminuído utilizando-se escala de notas, em vez de contagem de indivíduos, que são muitos e diminutos. E, por fim, utilizar a relação existente entre o número de colônias e o percentual de plantas atacadas favoreceria uma maior agilidade às decisões de manejo desta praga.

\section{Conclusões}

A unidade amostral para o pulgão do algodoeiro A. gossypii é a primeira folha totalmente expandida, a partir do ápice.

A relação entre o número de colônias e o percentual de plantas atacadas é dada pelo modelo linear.

\section{Agradecimento}

Os autores agradecem à CAPES pelo financiamento desta pesquisa.

\section{Referências}

ALCÂNTARA, E.; MORAES, J. C.; ANTONIO, A. Efeito de indutores da resistência e cultivares de algodão no comportamento de Aphis gossypii. Revista Ciência Agronômica, Fortaleza, v. 41, p. 619-624, 2010.

ANDRADE, L. H. et al. Efeito repelente de azadiractina e óleos essenciais sobre Aphis gossypii Glover (Hemiptera: Aphididae) em algodoeiro. Ciência Agronômica, Fortaleza, v. 44, p. 628-634, 2013.

ANDRADE, L. H. et al. Effects of botanical insecticides on the instantaneous population growth rate of Aphis gossypii Glover (Hemiptera: Aphididae) in cotton. Acta Scientiarum, Maringá, v. 34, p. 119-124, 2012.

ARAUJO, L. H. A. Novos sistemas de tomada de decisão a serem usados no manejo do pulgão Aphis gossypii (Glover, 1877) (Hemiptera: Aphididae) na cultura do algodoeiro. $89 \mathrm{f}$. Tese
(Doutorado em Agronomia)-Universidade Federal da Paraíba, Areia, 2009.

BARROS, R. et al. Efeitos da Adubação Nitrogenada em Algodoeiro sobre a Biologia de Aphis gossypii Glover (Hemiptera: Aphididae). Neotropical Entomology, Londrina, v. 36, p. 752-758, 2007.

BORTOLI, S. A. et al. Tabelas de vida de Orius insidiosus (say, 1832) (Hemiptera: Anthocoridae) predando Aphis gossypii Glover, 1877 (Hemiptera: Aphididae) em diferentes cultivares de algodoeiro. Arquivos do Instituto Biológico, São Paulo, v. 75, n. 2, p. 203-210, 2008.

BORTOLI, S. A.; VACARI, A. M.; FERNANDES, M. C.; BORTOLI, C. P.; BORTOLI, S. L. P.; RAMALHO, D. G. Efeito do espaçamento e de Bacillus thuringiensis Berliner sobre Alabama argillacea (Hübner), Aphis gossypii Glover e inimigos naturais no algodoeiro. Comunicata Scientiae, Teresina, v. 6, n. 2, p. 202-211, 2015.

CALCAGNOLO, G.; SAUER, H. F. G. A influência do ataque dos pulgões na produção do algodão (Aphis gossypii, Glover 1876, Hom. Aphididae). Arquivos do Instituto Biológico, São Paulo, v. 21, p. 85-89, 1954.

COSTA, L. L. et al. Diversidade e abundância de artrópodes predadores associados a diferentes cultivares de algodoeiro. Pesquisa Agropecuária Tropical, Goiânia, v. 40, p. 483-490, 2010.

FERNANDES, F. S. et al. Within-plant distribution of cotton aphid (Hemiptera: Aphididae) in cotton cultivars with colored fibers. Anais da Academia Brasileira de Ciências, Rio de Janeiro, v. 84, n. 3, p. 707-719, 2012.

FERNANDES, F. S.; RAMALHO, F. S.; NASCIMENTO JUNIOR, J. L.; MALAQUIAS, J. B.; NASCIMENTO, A. R. B.; SILVA, C. A. D; ZANUNCIO, J. C. Within-plant distribution of cotton aphids, Aphis gossypii Glover (Hemiptera: Aphididae), in $\mathrm{Bt}$ and non- $\mathrm{Bt}$ cotton fields. Bulletin of Entomological Research, Brisbane, v. 102, p. 1-9, 2011.

FURTADO, R. F. et al. Susceptibilidade de cultivares de Gossypium hirsutum L. r. latifolium Hutch a Aphis gossypii Glover. Revista Ciência Agronômica, Fortaleza, v. 40, p. 461-464, 2009.

FURTADO, R. F.; SILVA, F. P.; BLEICHER, E. Flutuação populacional de pulgão e cochonilha em cultivares diferentes de algodoeiro herbáceo. Revista Ciência Agronômica, Fortaleza, v. 38, p. 264-269, 2007.

GOUVEA, A.; BERTOLDO, A.; ALVES, L. F. A. Plano de amostragem presença-ausência para Oligonychus yothersi (McGregor) (Acari: Tetranychidae) na cultura da erva-mate. Neotropical Entomology, Londrina, v. 36, p. 583-586, 2007.

LI, Z. Q.; ZHANG, S; LUO J. Y.; WANG, C.Y.; LV L. M.; DONG, S. L.; CUI, J. J. Ecological adaption analysis of the cotton aphid (Aphis gossypii) in different phenotypes by transcriptome comparison. Plos One, San Francisco, n. 8, v. 12, 2013.

MICHELOTTO, M. D.; BUSOLI, A. C. Caracterização da transmissão do vírus do mosaico-das-nervuras do algodoeiro pelo pulgão Aphis gossypii com relação à persistência e ao tempo necessário para inoculação. Bragantia, Campinas, v. 66, p. 441-447, 2007.

MULLINS, G. L.; BURMESTER, C. H. Relation of growth and development to mineral nutrition. In: STEWART, J.; OOSTERHUIS, D. M.; HEITHOLT, J. J.; MAUNEY, J. R. (Eds.). Physiology of cotton. New York: Elsevier, 2010. cap. 9, p. 97-105.

PEDIGO, L. P.; RICE, M. E. (Ed.). Entomology and pest management. New Jersey: Upper Saddle River, 2009. 784 p.

PESSOA, L. G. et al. Aspectos da biologia de Aphis gossypii Glover, 1877 (Hemiptera: Aphididae) em quatro cultivares de algodoeiro 
em laboratório. Ciência e Agrotecnologia, Lavras, v. 28, p. 235-239, 2004.

POWELL, G.; TOSH, C. R.; HARDIE, J. Host plant selection by aphids: behavioral, evolutionary, and applied perspectives. Annual Review of Entomology, Palo Alto, v. 51, p. 309-330, 2006.

SANTOS, W. J. Manejo das pragas do algodão com destaque para o cerrado brasileiro. In: Freire, E. C. (Ed.). Algodão no cerrado do Brasil. Brasília: ABRAPA, 2007. p. 403-478.

SILVA, A. M. et al. Distribuição espacial de pulgões Aphis gossypii (Glover, 1877) em plantas de algodoeiro. In: CONGRESSO BRASILEIRO DO ALGODÃO, 6., 2007, Uberlândia. Anais... Salvador: $1 C D$.

SILVA, A. M.; FERNANDES, M. G.; DEGRANDE, P. E. Distribuição vertical de pulgões Aphis gossypii (Glover, 1877) em plantas de algodoeiro. In: CONGRESSO BRASILEIRO DO ALGODÃO, 5. 2005, Salvador. Anais... Fortaleza, 1CD.

SMITH, J. P.; SHEPARD, B. M. A binomial sequential sampling plan using a composite threshold for caterpillar management in fresh market collard. Journal of Agricultural and Urban Entomology, Columbia, v. 21, p. 171-184, 2004.
SPRENKEL, R. K. Cotton pest monitoring manual for Florida. Florida: IFAS Extension University of Florida, 2008. 24 p.

SUJI, E. R. et al. Impacto do algodoeiro Bt na dinâmica populacional do pulgão-do-algodoeiro em casa de vegetação. Pesquisa Agropecuária Brasileira, Brasília, v. 43, n. 10, p. 1251-1256, 2008.

TAKIMOTO, J. K. et al. Alterações anatômicas em algodoeiro infectado pelo vírus da doença azul. Bragantia, Campinas, v. 68, p. 109-116, 2009.

TORRES, L. C. et al. Biologia e não-preferência para oviposição por Bemisia tabaci (Gennadius) biótipo B (Hemiptera: Aleyrodidae) em cultivares de algodoeiro. Neotropical Entomology, Londrina, v. 36, n. 3, p. 445-453, 2007.

WILSON, L. T. et al. Within-plant distribution of spider mites (Acari: Tetranychidae) on cotton: a developing implementable monitoring program. Environmental Entomology, Annapolis, v. 12, p. 128-134, 1983. 